\title{
On the breathing frequencies computation using the Reissner and the Mindlin model
}

\author{
L. Palermo Jr. \\ Faculty of Civil Engineering, Architecture and Urban Design, \\ State University of Campinas, Brazil
}

\begin{abstract}
The breathing frequencies of plates were computed using the frequency domain solution in conjunction with direct boundary integral equations (DBIEs) for plate bending including the shear deformation effect and the influence of rotatory inertia according to Reissner and Mindlin models. The formulation for the Reissner model takes into account the linearly weighted average effect of the normal stress component in the thickness direction with reference to that for the Mindlin model. Furthermore, the irrotational approach on the bending model containing the shear deformation effect to obtain the classical model is revisited taking into account the Reissner and the Mindlin model. Flexural and breathing frequencies for both models including the shear deformation effect and the influence of rotatory inertia were obtained and compared with available results in the literature to show the accuracy of the formulation.

Keywords: natural frequencies, breathing frequencies, frequency domain solution, classical bending model, reissner bending model, mindlin bending model, rotatory inertia.
\end{abstract}

\section{Introduction}

The classical model was the first and well-known approach derived from the three-dimensional theory of elasticity for solid bodies to study plate-bending problems. The model is widely employed when global responses are required but it is not efficient even in static analyses when stresses are assessed in the edge zone of the plate or around holes that have a diameter not larger than eight times the plate thickness [1]. The discrepancy in results of dynamic analyses with the classical model with reference to the three-dimensional theory appears when 
wavelengths become less than ten times the plate thickness and an inconsistency is verified for wavelengths approaching to zero when a limit for the velocity of propagation cannot be established [2]. The plate-bending model including the shear deformation effect proposed by Reissner [3] has fixed the main shortcomings verified in the classical model. An assumed distribution for stresses on the plate thickness and a fixed value for the parameter related to the shear deformation in the constitutive equations were the main features observed in the Reissner model. A similar model for plate-bending including the shear deformation effect but using an assumed distribution for strains with reference to the mid-surface of the plate was presented by Mindlin [2] to perform dynamic analyses. A correction of the parameter related to the shear deformation according to the Poisson ratio was proposed as a result from the analysis of the propagation of straight-crested waves into an infinite domain. On the other hand, a fixed value for this parameter and very close to Reissner's value was obtained when a particular solution was introduced by Mindlin to represent one for the circular frequency of the first anti-symmetric mode of the thickness-shear vibration in a three-dimensional model. Years later, Mindlin has shown in the study of flexural vibration of rectangular plates [4] that three types of vibration modes could be identified when the shear deformation effect was considered: the flexural, the thickness-shear and the thickness-twist mode. The thickness-twist mode is similar to the wave motion employed in [2] to get the fixed value for the parameter related to the shear deformation. The frequencies obtained with the thickness-twist mode were called breathing frequencies by Levinson [5].

The static or the frequency domain solutions have been employed for dynamic analyses of plates using the Boundary Element Method (BEM) beyond special formulations applied to specific problems [6]. The harmonic analyses of plates in this paper were carried out for Reissner and Mindlin models employing the frequency domain fundamental solution presented in [7,8], which was not the same used in [9] where the formulation was only based on the real part of the solution and a limit for angular frequencies had to be introduced. The effect of the shear deformation and the influence of rotatory inertia can be considered alone or coupled in the present formulation. The linearly weighted average effect of the normal stress component in the thickness direction of the Reissner model is considered in the DBIE as an additional domain integral when distributed loads are considered. Analyses according to the classical model with or without the influence of rotatory inertia can be obtained when the shear deformation effect is disregarded in the formulations for both models.

\section{Direct boundary integral equations}

The equations of motion in time-harmonic problems for an infinitesimal plate element under a transverse distributed loading $\mathrm{q}\left(\mathrm{t}, \mathrm{x}_{\mathrm{i}}\right)$ are next written with Latin indices taking values $\{1,2$ and 3$\}$ and Greek indices taking values $\{1,2\}$ :

$$
\mathrm{M}_{\alpha \beta, \beta}-\mathrm{Q}_{\alpha}=\frac{\rho \mathrm{h}^{3}}{12} \frac{\partial^{2} \psi}{\partial \mathrm{t}^{2}}
$$




$$
\mathrm{Q}_{\alpha, \alpha}+\mathrm{q}=\rho \mathrm{h} \frac{\partial^{2} \mathrm{w}}{\partial \mathrm{t}^{2}}
$$

The plate has a uniform thickness $\mathrm{h}$ and the mass density $\rho$. The transverse acceleration $\partial^{2} \mathrm{w} / \partial \mathrm{t}^{2}$ and the angular acceleration $\partial^{2} \psi_{\alpha} / \partial \mathrm{t}^{2}$ are functions of the deflection $\mathrm{W}$ and the components of changes of slope $\psi_{\alpha}$ (plate rotations), respectively. The constitutive relations are next written using a unified notation for the Reissner and the Mindlin models:

$$
\begin{gathered}
\mathrm{M}_{\alpha \beta}=\mathrm{D} \frac{1-v}{2}\left(\psi_{\alpha, \beta}+\psi_{\beta, \alpha}+\frac{2 v}{1-v} \psi_{\gamma, \gamma} \delta_{\alpha \beta}\right)+\mathrm{RE} \\
\mathrm{Q}_{\alpha}=\mathrm{D} \frac{1-v}{2} \lambda^{2}\left(\psi_{\alpha}+\mathrm{w}_{, \alpha}\right)
\end{gathered}
$$

with

$$
\begin{gathered}
\lambda^{2}=12 \frac{\kappa^{2}}{h^{2}} \\
R E=\frac{v}{\lambda^{2}(1-v)} q
\end{gathered}
$$

$\mathrm{D}$ is the flexural rigidity, $v$ is the Poisson ratio, RE is zero for the Mindlin model because it corresponds to the linearly weighted average effect of the normal stress component in the thickness direction. The shear parameter $\kappa^{2}$ is equal to $5 / 6$ and $\pi^{2} / 12$ for the Reissner and the Mindlin models, respectively. An alternate value for $\kappa^{2}$ according to the Poisson ratio can be calculated with the following equation $[2,8]$ :

$$
4 \sqrt{\left(1-\alpha \kappa^{2}\right)\left(1-\kappa^{2}\right)}=\left(2-\kappa^{2}\right)^{2}
$$

with

$$
\alpha=\frac{1-2 v}{2(1-v)}
$$

The static like form of the equations of motion for harmonic problems and the unified notation for the constitutive equations carry to a unified DBIE for the Reissner and the Mindlin model similar to that presented by Weeën in [10] but used in conjunction with the elastodynamic fundamental solution instead:

$$
\frac{1}{2} \mathrm{C}_{\mathrm{ij}} \mathrm{u}_{\mathrm{j}}+\oint_{\Gamma}\left(\mathrm{T}_{\mathrm{j}}^{\mathrm{i}} \mathrm{u}_{\mathrm{j}}-\mathrm{U}_{\mathrm{j}}^{\mathrm{i}} \mathrm{t}_{\mathrm{j}}\right) \mathrm{d} \Gamma=\iint_{\Omega}\left(\mathrm{U}_{3}^{\mathrm{i}} \mathrm{q}-\mathrm{U}_{\alpha, \alpha}^{\mathrm{i}} R E\right) \mathrm{d} \Omega
$$

where $\mathrm{u}_{\alpha}$ is $\psi_{\alpha}, \mathrm{u}_{3}$ is $\mathrm{w}, \mathrm{t}_{\alpha}$ is the product $\mathrm{M}_{\alpha \beta} \cdot \mathrm{n}_{\beta}, \mathrm{t}_{3}$ is the product $\mathrm{Q}_{\alpha} \cdot \mathrm{n}_{\alpha} \cdot \mathrm{U}_{\mathrm{j}}^{\mathrm{i}}$ represents the rotation $(\mathrm{j}=1,2)$ or the deflection $(\mathrm{j}=3)$ due to a unit couple $(\mathrm{i}=1,2)$ or a unit point force $(\mathrm{i}=3) . \mathrm{C}_{\mathrm{ij}}$ is an element of the matrix $\mathrm{C}$ related to the boundary at the source point and it is a diagonal matrix when a smooth boundary is considered: $\mathrm{c}_{11}$ and $\mathrm{c}_{22}$ are equal to $\left(1+0.5 \mathrm{RS}_{0}{ }^{4}\right), \mathrm{c}_{33}$ is equal to 1 . C becomes the Kronecker delta when the rotatory inertia effect is disregarded. The integrand of the domain integral in equation (9) contains the factor RE related to the linearly weighted average effect of the normal stress component in the thickness direction, which should be cut off for analyses using the Mindlin model. 
The fundamental solution was presented in $[7,8]$ in terms of Hankel functions of the first kind of order zero or using modified Bessel functions with complex arguments. The vector of plate rotations $\left(\psi_{\alpha}\right)$ in the fundamental solution was written in terms of its scalar $\left(\phi_{\alpha}\left(\mathrm{x}_{\alpha}, \omega\right)\right)$ and vector potentials $\left(0,0, \mathrm{H}\left(\mathrm{x}_{\alpha}, \omega\right)\right)$ :

$$
\psi_{\alpha}=\frac{\partial}{\partial \mathrm{x}_{\alpha}}\left[\phi_{1}\left(\delta_{1} \mathrm{r}\right)+\phi_{2}\left(\delta_{2} \mathrm{r}\right)\right]+\mathrm{e}_{3 \alpha \beta} \frac{\partial}{\partial \mathrm{x}_{\beta}}\left[\mathrm{H}\left(\delta_{3} \mathrm{r}\right)\right]
$$

$\mathrm{e}_{3 \alpha \beta}$ is the permutation symbol and the following relations were used in terms of the rotatory inertia $(\mathrm{R})$, the shear deformation $(\mathrm{S})$ and the classical dynamic factors for plates $\left(\delta_{0}{ }^{4}\right)$ :

$$
\begin{gathered}
\delta_{1}^{2}=\frac{1}{2} \delta_{0}^{4}\left(\mathrm{R}+\mathrm{S}+\sqrt{(\mathrm{R}-\mathrm{S})+\frac{4}{\delta_{0}^{4}}}\right) ; \quad \delta_{2}^{2}=\frac{1}{2} \delta_{0}^{4}\left(\mathrm{R}+\mathrm{S}-\sqrt{(\mathrm{R}-\mathrm{S})+\frac{4}{\delta_{0}^{4}}}\right) \\
\delta_{3}^{2}=\frac{2}{1-v}\left(\mathrm{R} \delta_{0}^{4}-\frac{1}{\mathrm{~S}}\right)=\lambda^{2}\left(\mathrm{RS} \delta_{0}^{4}-1\right) \\
\mathrm{R}=\frac{\mathrm{h}^{2}}{12} ; \quad \mathrm{S}=\frac{1}{\lambda^{2}} \frac{2}{1-v} ; \quad \delta_{0}^{4}=\frac{\omega^{2} \rho \mathrm{h}}{\mathrm{D}}
\end{gathered}
$$

The deflection w was related to the scalar potential functions $\left(\phi_{\alpha}\right)[7,8]$ :

$$
\mathrm{w}=-\left(\beta_{1}+1\right) \phi_{1}\left(\delta_{1} \mathrm{r}\right)-\left(\beta_{2}+1\right) \phi_{2}\left(\delta_{2} \mathrm{r}\right)
$$

and

$$
\beta_{1}=\mathrm{S}\left(\delta_{1}^{2}-\delta_{0}^{4} \mathrm{R}\right) ; \quad \beta_{2}=\mathrm{S}\left(\delta_{2}^{2}-\delta_{0}^{4} \mathrm{R}\right)
$$

The expressions for the scalar and the vector potential functions of the fundamental solution are next written using modified Bessel functions with complex arguments and according to singular loads used:

a) The solution for the unit point force $\left(\mathrm{F}_{3}\right)$ :

$$
\begin{aligned}
& \phi_{1}=\frac{1}{2 \pi \mathrm{D}} \frac{-\mathrm{K}_{0}\left(\mathrm{i}_{1} \mathrm{r}\right)}{\sqrt{\delta_{0}^{8}(\mathrm{R}-\mathrm{S})+4 \delta_{0}^{4}}}=\frac{1}{2 \pi \mathrm{D}} \frac{-\mathrm{K}_{0}\left(\mathrm{i} \delta_{1} \mathrm{r}\right)}{\left(\delta_{1}^{2}-\delta_{2}^{2}\right)} ; \\
& \phi_{2}=\frac{1}{2 \pi \mathrm{D}} \frac{\mathrm{K}_{0}\left(\mathrm{i} \delta_{2} \mathrm{r}\right)}{\sqrt{\delta_{0}^{8}(\mathrm{R}-\mathrm{S})+4 \delta_{0}^{4}}}=\frac{1}{2 \pi \mathrm{D}} \frac{\mathrm{K}_{0}\left(\mathrm{i} \delta_{2} \mathrm{r}\right)}{\left(\delta_{1}^{2}-\delta_{2}^{2}\right)} \\
& \mathrm{H}=0
\end{aligned}
$$

b) The solution for the unit couple in the $\alpha$ direction $\left(\mathrm{F}_{\alpha}\right)$ :

$$
\begin{aligned}
& \phi_{1}=\frac{1}{2 \pi \mathrm{D}} \frac{-1}{\left(\delta_{1}^{2}-\delta_{2}^{2}\right)} \frac{\partial}{\partial \mathrm{x}_{\alpha}}\left[\mathrm{K}_{0}\left(\mathrm{i} \delta_{1} \mathrm{r}\right)\right] ; \quad \phi_{2}=\frac{1}{2 \pi \mathrm{D}} \frac{1}{\left(\delta_{1}^{2}-\delta_{2}^{2}\right)} \frac{\partial}{\partial \mathrm{x}_{\alpha}}\left[\mathrm{K}_{0}\left(\mathrm{i} \delta_{2} \mathrm{r}\right)\right] ; \\
& \mathrm{H}=\mathrm{e}_{3 \alpha \beta} \frac{\mathrm{S}}{2 \pi \mathrm{D}} \frac{\partial}{\partial \mathrm{x}_{\beta}}\left[\mathrm{K}_{0}\left(\mathrm{i} \delta_{3} \mathrm{r}\right)\right]
\end{aligned}
$$

The solution becomes equal to that obtained for the classical bending model [11] when the shear deformation $(\mathrm{S})$ and rotatory inertia $(\mathrm{R})$ effects are set equal to zero. Furthermore, the plate rotations turn directly defined from the deflection function derivatives as a result from the classical model because the constants $\beta_{\alpha}$ and the function $\mathrm{H}$ are equal to zero in the absence of the shear deformation 
effect (S). The field decomposition can be introduced in the plate rotations and their derivatives of the constitutive equations $(3,4)$ as it was done to the plate rotations of the fundamental solutions (equation (10)). After the introduction of the scalar and the vector potential functions in the constitutive equations, the equations of the classical model can be obtained when the function of the vector potential field is set equal to zero and the scalar potential function uses the deflection function (w). It can be also extended to the DBIE yielding a boundary formulation based on the classical model hypotheses $[7,8]$.

The domain integral including the Reissner effect (RE) of equation (9) can be simplified when the field decomposition is introduced in rotations $\mathrm{U}_{\alpha}^{\mathrm{i}}$ according to equation (10) and employing the first Green identity to get the final relation:

$$
\iint_{\Omega} \mathrm{U}_{\alpha, \alpha}^{\mathrm{i}} \mathrm{REd} \Omega=\oint_{\Gamma}\left(\phi_{1}+\phi_{2}\right)_{{ }_{\mathrm{n}}}^{\mathrm{i}} \mathrm{REd} \Gamma-\iint_{\Omega}\left(\phi_{1}+\phi_{2}\right)_{, \alpha}^{\mathrm{i}} \mathrm{RE}_{, \alpha} \mathrm{d} \Omega
$$

The result from equation (13) shows the linearly weighted average effect of the normal stress component in the thickness direction (RE) is only multiplied by the scalar potential function or the irrotational component of the rotation field. The effect reduces to an additional boundary integral when a uniform loading (q) is considered because it is directly related to RE according to equation (6).

The irrotational approach on the present formulation to perform analyses according to the classical bending model is similar to that shown in [12] for static loads using the Mindlin model. The first step is writing the DBIE (9) with reference to the normal and the tangential directions (nt) at the boundary. It is not a necessary step but the approach can be easily visualized in this way:

$$
\begin{aligned}
& \frac{1}{2} C_{i j} u_{j}+\oint_{\Gamma}\left(Q_{n}^{i} w+M_{n}^{i} \theta_{n}+M_{n t}^{i} \theta_{t}-Q_{n} w^{i}-M_{n} \theta_{n}^{i}-M_{n t} \theta_{t}^{i}\right) d \Gamma=\ldots \\
& \ldots=\iint_{\Omega}\left[w^{i} q+\left(\phi_{1}+\phi_{2}\right)_{, \alpha}^{i} R E_{, \alpha}\right] d \Omega-\oint_{\Gamma}\left(\phi_{1}+\phi_{2}\right)_{, n}^{i} R E d \Gamma
\end{aligned}
$$

With i relating to the fundamental solution used, corresponding to a unit point load ( $\mathrm{i}=3$ ) or a unit couple ( $\mathrm{i}=1$ or 2$)$; $\mathrm{u}_{\mathrm{j}}$ represents the deflection $(\mathrm{j}=3)$ or the rotation (j=1 or 2). $\theta_{n}$ and $\theta_{t}$ stand for $\psi_{n}$ and $\psi_{t}$, respectively. $M_{n}$ and $M_{n t}$ are the bending and twisting moments, respectively. $\mathrm{Q}_{\mathrm{n}}$ is the distributed shear.

The irrotational approach is equivalent to disregarding the field component related to the vector potential function. The simplification in the presented formulation corresponds to set the shear deformation effect (S) equal to zero in the fundamental solution and assuming plate rotations $\theta_{\mathrm{n}}$ and $\theta_{\mathrm{t}}$ equal to $-\mathrm{W}_{\mathrm{n}, \mathrm{n}}$ and $-\mathrm{w}_{\mathrm{t}}$, respectively, as well as releasing the twisting moments $\mathrm{M}_{\mathrm{nt}}$ on the boundary $\left(\mathrm{M}_{\mathrm{nt}}=0\right)$. The classical hypotheses are satisfied with these assumptions. The resultant DBIE allows dynamic analyses of plates by applying the rotatory inertia correction on the classical model and is analogous to the well-known DBIE used for static analyses within classical plate theory, including an additional degree of freedom for the tangential boundary rotation [12]. 


$$
\frac{1}{2} \mathrm{C}_{\mathrm{ij}} \mathrm{u}_{\mathrm{j}}+\oint_{\Gamma}\left(\mathrm{Q}_{\mathrm{n}}^{\mathrm{i}} \mathrm{w}-\mathrm{M}_{\mathrm{n}}^{\mathrm{i}} \mathrm{w}_{, \mathrm{n}}-\mathrm{M}_{\mathrm{nt}}^{\mathrm{i}} \mathrm{w}_{, \mathrm{t}}-\mathrm{Q}_{\mathrm{n}} \mathrm{w}^{\mathrm{i}}+\mathrm{M}_{\mathrm{n}} \mathrm{w}_{, \mathrm{n}}^{\mathrm{i}}\right) \mathrm{d} \Gamma=\iint_{\Omega} \mathrm{w}^{\mathrm{i}} \mathrm{q} \mathrm{d} \Omega .
$$

The DBIE for the classical bending model without the rotatory inertia correction is obtained when the parameter $\mathrm{R}$ is set equal to zero in the elastodynamic solution used in equation (15).

The absence of the shear deformation effect $(\mathrm{S}=0)$ corresponds to assume the shear parameter $\kappa^{2}$ approaching to infinite (or $\lambda^{2}$, see equation 5) and an orthotropic material replaces the actual material of the plate, supposed to be isotropic, as explained by Timoshenko and Woinowsky-Krieger [1] on the classical hypotheses. The factor RE was not included in equation (15) because it contains the shear parameter $\lambda^{2}$. The effect (RE) approaches to zero when the parameter $\left(\lambda^{2}\right)$ approaches to infinite, which shows that an irrotational approach on the Reissner model carries to the same result obtained with the Mindlin model.

\section{Numerical example}

The dynamic analyses of plates considering the Reissner and the Mindlin models show similar results and the difference appeared in values obtained for displacements related to the linearly weighted average effect of the normal stress component in the thickness direction, which is dependent to the pressure of the surface loading. The difference in values for displacements is shown in Figure 1 using the frequency response of a square plate simply supported on all sides and $30 \mathrm{~cm}$ for thickness (h). A uniform loading (q) equal to $150 \mathrm{~N} \cdot \mathrm{m}^{-2}$ was applied over the plate surface which side (a) is equal to $50 \mathrm{~cm}$, Young's modulus was $206.9 \mathrm{GPa}$, the Poisson ratio ( $v$ ) was 0.3 , the mass density $(\rho)$ was $7860 \mathrm{~kg} . \mathrm{m}^{-3}$. Linear shape functions were employed to approximate displacements and efforts in the boundary elements. All nodal parameters were positioned at the ends of the elements and the collocation points were placed on the boundary. The collocation points were shifted to the interior of the element at a distance of a quarter of its length from the end, for discontinuous boundary elements, and shifted to the center of the element for continuous boundary elements. The integrations were performed with analytical expressions for the case of collocation points belonging to the boundary element and the Gauss-Legendre quadrature for other cases.

The values of natural frequencies were not changed independently of the adopted model. The natural frequency could be identified in the harmonic excitation using the value of a displacement (deflection or rotation) at an internal point but others features could be employed as the reference parameter, such as the phase angle or the determinant of the system matrix. A first evaluation with 20 incremental steps was carried out in this study to find the interval of interest. The interval to be focused, containing 2 incremental steps, was picked up when the sign of the real part of the deflection changed as shown in Figure 1. The focused interval was analyzed again with a refinement equals to 40 incremental steps and two peaks with opposite signs could be identified in the response curve 


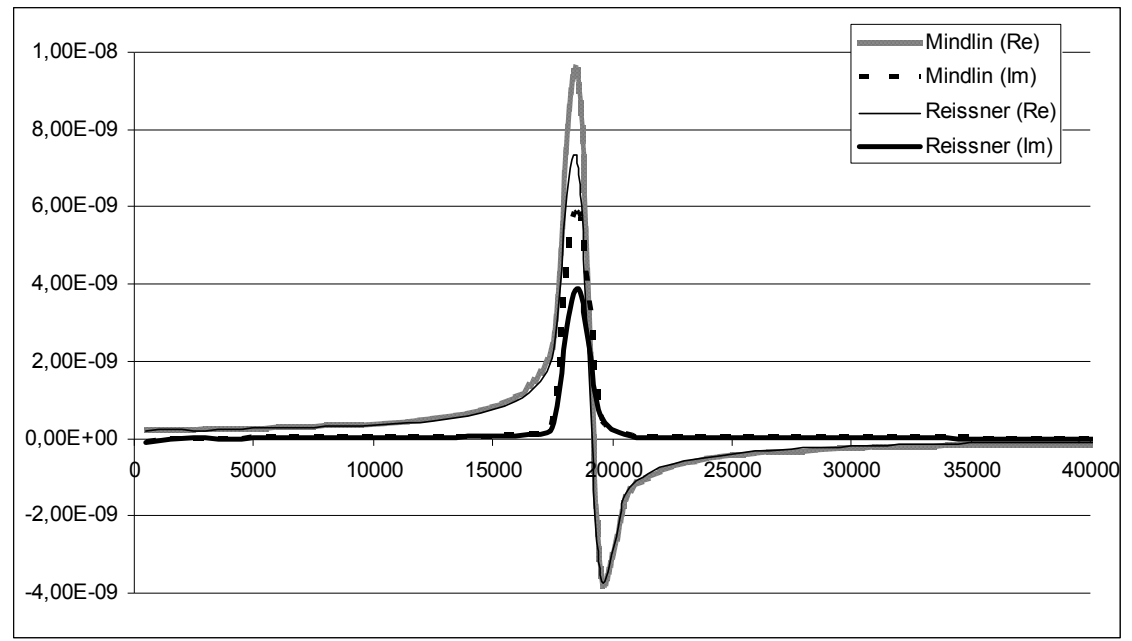

Figure 1: Responses using the Reissner and the Mindlin model for $\mathrm{h} / \mathrm{a}=0.6$ and including the rotatory and the shear deformations effects.

Table 1: Angular frequencies of the flexural mode ( $\mathrm{rad} / \mathrm{s})$.

\begin{tabular}{|c|c|c|c|c|c|}
\hline \multirow{2}{*}{ Ratio h/a } & Lim. Freq. & \multirow{2}{*}{ Elasticity [5] } & Mindlin [4] & \multicolumn{2}{|c|}{ Equation (14) } \\
\cline { 2 - 4 } & $\lambda \mathrm{c}_{\mathrm{s}}[8]$ & & & Mindlin & Reissner \\
\hline 0.05 & 199923 & 3016 & 3049 & 3015 & 3015 \\
\hline 0.1 & 99961 & 5906 & 5918 & 5825 & 5825 \\
\hline 0.2 & 49980 & 10880 & 10820 & 10380 & 10380 \\
\hline 0.4 & 24990 & 17315 & 17073 & 15760 & 15760 \\
\hline 0.6 & 16660 & 20734 & 20306 & 18910 & 18910 \\
\hline 0.8 & 12495 & 22653 & 22079 & 21480 & 21480 \\
\hline 1.0 & 9996 & 23805 & 23125 & 23570 & 23570 \\
\hline
\end{tabular}

of the real part of the deflection. The frequency responses were studied for several ratios $(\mathrm{h} / \mathrm{a})$ of the plate simply supported on all sides and using the hard restraint condition. The first natural frequencies obtained by using the numerical implementation for the Reissner and the Mindlin model are listed in Table 1 and compared to values available in the literature, obtained by the Mindlin model [4] and employing three-dimensional elasticity theory [5].

The next frequencies could be identified by using the same loading but increasing the scanned range. It is important to note the natural frequencies can be easily identified when their vibration modes are not damped due to a particular loading condition or the internal point position, as explained in [13]. The vibration modes of natural frequencies shown in Table 1 have curvatures with same sign and are related to flexural vibration modes [4]. 
The flexural and the thickness-shear modes are related to the scalar potential field whereas the thickness-twist mode is related to the vector potential field [4]. The rotation field of the thickness-twist mode is similar to that obtained from an anticlastic vibration surface, which contains curvatures with opposite signs [1]. Levinson [5] called the natural frequency of the thickness-twist mode as breathing frequency. The distributed loading (q) was set equal to zero in the numerical implementation to scan the first breathing frequency and the DBIEs (14) turn the same for the Reissner or the Mindlin model. A bending moment in the normal direction was applied on the node placed at the center of each side of the plate. The moments had same sign on opposite edges and opposite signs on adjacent edges. The domain was contained in the intervals $0<\mathrm{x}_{1}<0.5$ and $0<\mathrm{x}_{2}<0.5$, and the value of the rotation $\psi_{2}$ at the point $(0.1875,0.1875)$ was used to scan the natural frequencies. The breathing frequencies listed in Table 2 were obtained with the influence of rotatory inertia. The values obtained for breathing frequencies using the Mindlin model and the three-dimensional elasticity theory are close from medium to high ratios (h/a) but they are divergent for low ratios [5] where a limiting value for the frequency is obtained in the three dimensional elasticity theory as shown in Table 2 .

Figure 2 shows an example of the frequency response curves to find breathing frequencies for ratios $(\mathrm{h} / \mathrm{a})$ in the range $(0.4,1.0)$. There are few natural frequencies lower than the first breathing frequency for plates with ratio $(\mathrm{h} / \mathrm{a})$ in that range. The loading condition with bending moments in the same directions of rotations of the first thickness-twist vibration mode was efficient to obtain the requested frequencies. On the other hand, there are several natural frequencies lower than the first breathing frequency when the ratio (h/a) is 0.2 or lower and several peaks with opposite signs are identified in the frequency response as shown in Figure 3 and the lower frequencies are related to flexural vibration modes. In spite of a frequency close the value $103870 \mathrm{rad} / \mathrm{s}$ appeared in the analysis of Figure 3, the visualization was not clear to identify the natural frequency without using theoretical values. It was the reason to do not include a value in Table 2 for ratios lower than 0.4 .

Table 2: $\quad$ Angular frequencies of the thickness-twist mode ( $\mathrm{rad} / \mathrm{s})$.

\begin{tabular}{|c|c|c|c|}
\hline Ratio h/a & Elasticity [5] & Mindlin [4] & Equation (14) \\
\hline 0.05 & 47750 & 400790 & - \\
\hline 0.1 & 47700 & 201880 & - \\
\hline 0.2 & 47460 & 103870 & - \\
\hline 0.4 & 46230 & 57415 & 53000 \\
\hline 0.6 & 42860 & 43693 & 42100 \\
\hline 0.8 & 37410 & 37729 & 36800 \\
\hline 1.0 & 33094 & 34263 & 33750 \\
\hline
\end{tabular}




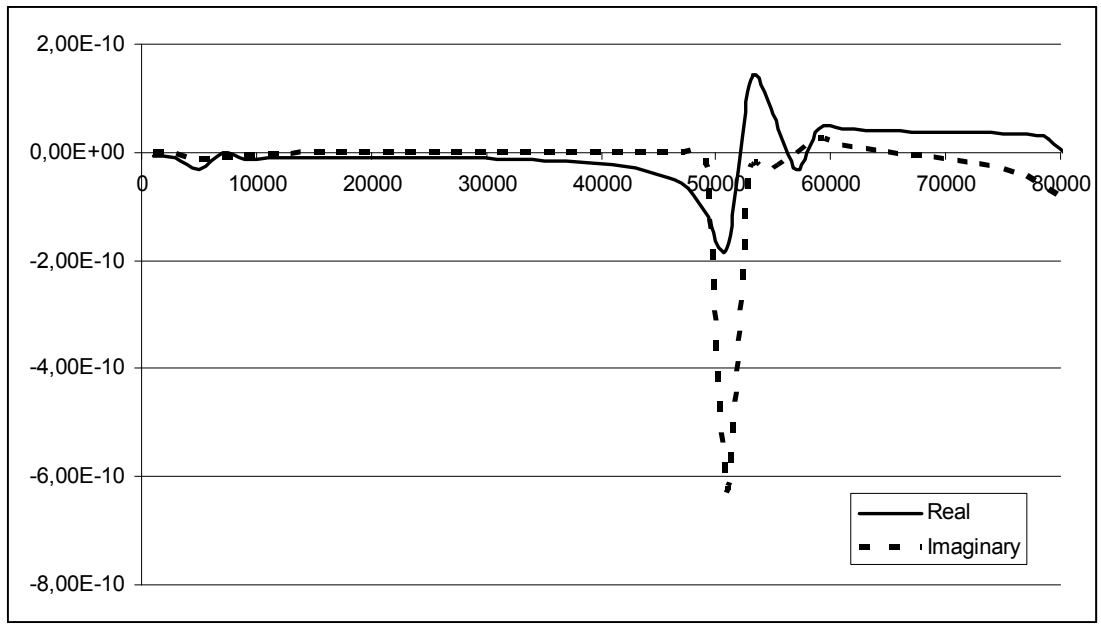

Figure 2: $\quad$ Rotation $\psi_{2}$ at $(0.1875,0.1875)$ for $\mathrm{h} / \mathrm{a}=0.4$.

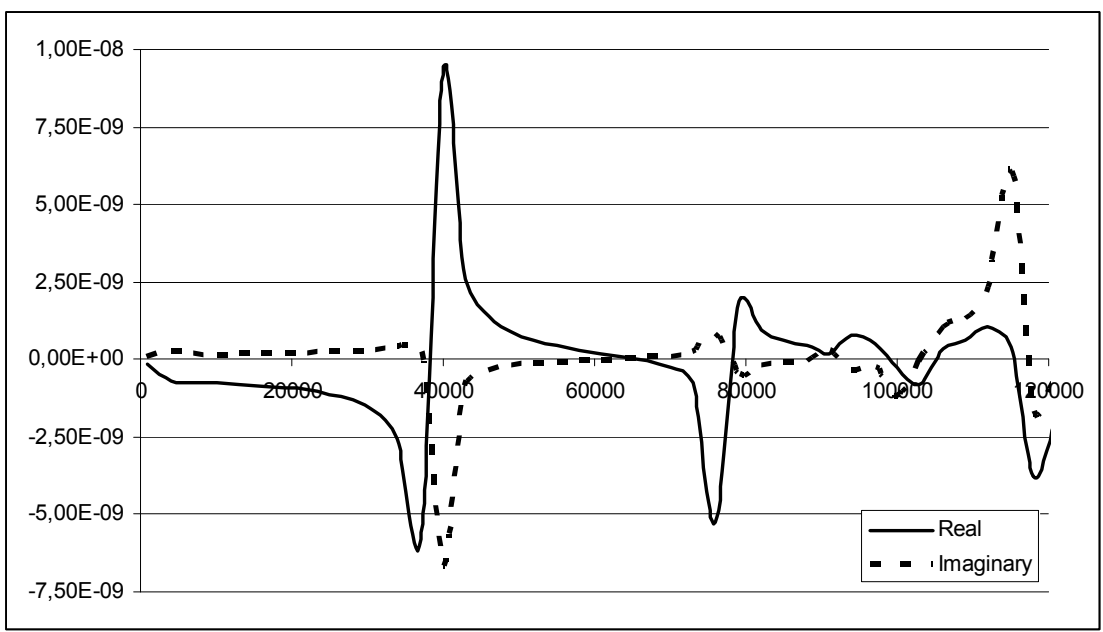

Figure 3: $\quad$ Rotation $\psi_{2}$ at $(0.1875,0.1875)$ for $\mathrm{h} / \mathrm{a}=0.2$.

\section{Conclusions}

The linearly weighted average effect of the normal stress component in the thickness direction of the Reissner model introduced a little reduction on the obtained deflection with reference to that using the Mindlin model. The difference can be significant or not according to the thickness value and the boundary conditions. It is important to note the natural frequencies were not changed according to Reissner's or Mindlin's model and the differences were identified in values for displacements. The existence of three types of vibration modes and corresponding frequencies for each mode, including those with high 
frequencies, justifies the present formulation employing modified Bessel functions of the second kind with complex arguments for a general treatment of vibration problems of plates. The limiting frequency $\lambda c_{s}[8]$, where $c_{s}$ is the equivolumial wave velocity, that defines complex or real arguments of modified Bessel functions were exceeded in the natural frequencies of the Table 1 for ratios in the range $(0.6,1.0)$.

\section{Acknowledgements}

The author is grateful to CNPq and FAPESP for support for the development of research on plates.

\section{References}

[1] Timoshenko, S. P., Woinowsky-Krieger, S., Theory of Plates and Shells, McGraw-Hill Book Company, New York, 2nd Ed., 1959

[2] Mindlin, R.D., Influence of rotatory inertia and shear on flexural motions of isotropic elastic plates, Journal of Applied Mechanics, 1951.

[3] Reissner, E., The Effect of Transverse Shear Deformation on the Bending of Elastic Plates, Journal of Applied Mechanics, 1945.

[4] Mindlin, R.D., Schacknow A., Deresiewicz, H., Flexural vibrations of rectangular plates, Journal of Applied Mechanics, 23, 430-436, 1956.

[5] Levinson, M., Free vibrations of a simply supported rectangular plate: an exact elasticity solution, Journal of Sound and Vibration, 98(2), 289-298, 1985.

[6] Providakis CP, Beskos DE. Dynamic analysis of plates by the boundary elements. Appl Mech Rev.;52(7) ASME, 1999.

[7] Palermo Jr., L., On the fundamental solution to perform the dynamic analysis of Reissner-Mindlin's plates, Boundary Element Technology XV, Editors C.A. Brebbia, R.E. Dippery, 2003

[8] Palermo Jr., L., On the harmonic response of plates with the shear deformation effect using the elastodynamic solution in the boundary element method, Engineering Analysis with Boundary Elements, 2007.

[9] Antes, H., Static and Dynamic Analysis of Reissner-Mindlin Plates, Boundary Element Analysis of Plates and Shells, Editor: D.E. Beskos, Springer Series in Computational Mechanics Edited by S.N. Atluri, Springer-Verlag, 1991.

[10] Weeën, F., Application of the direct boundary element method to Reissner's plate model, International Journal for Numerical Methods in Engineering, 1982.

[11] Vivoli J, Filippi P. Eigenfrequencies of thin plates and layer potentials. J Acoust Soc Am; 55:562-7, 1974.

[12] Palermo Jr., L., Plate Bending analysis using the classical or the ReissnerMindlin models, Engineering Analysis with Boundary Elements, 2003.

[13] Warburton, G. B., The dynamical behavior of structures, Pergamon Press, Oxford, 1976. 